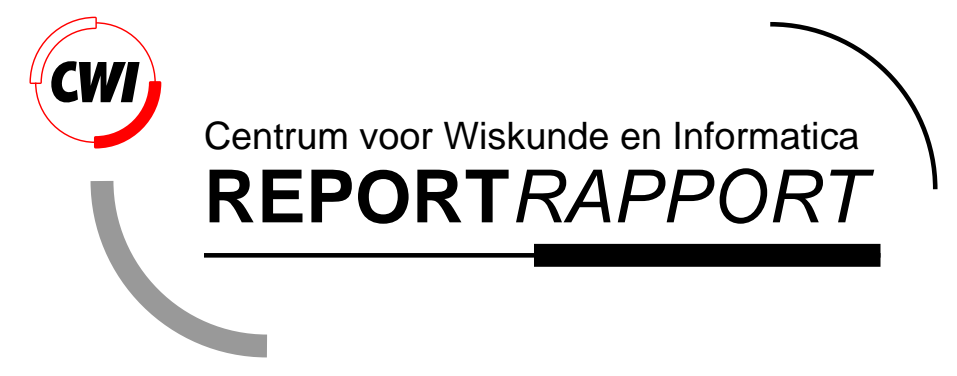

On multi-query optimization

R. Choenni M.L. Kersten, J.F.P. van den Akker and A. Saad

Computer Science/Department of Algorithmics and Architecture

CS-R9638 1996 
Report CS-R9638

ISSN 0169-118X

CWI

P.O. Box 94079

1090 GB Amsterdam

The Netherlands

$\mathrm{CWI}$ is the National Research Institute for Mathematics and Computer Science. CWI is part of the Stichting Mathematisch Centrum (SMC), the Dutch foundation for promotion of mathematics and computer science and their applications.

SMC is sponsored by the Netherlands Organization for Scientific Research (NWO). CWI is a member of ERCIM, the European Research Consortium for Informatics and Mathematics.

Copyright (C) Stichting Mathematisch Centrum P.O. Box 94079, 1090 GB Amsterdam (NL) Kruislaan 413, 1098 SJ Amsterdam (NL) Telephone +3120 5929333 Telefax +31205924199 


\title{
On Multi-Query Optimization
}

\author{
Sunil (R.) Choenni Martin L. Kersten Johan F.P. van den Akker \\ $C W I$, \\ P.O. Box 94079, 1090 GB Amsterdam, The Netherlands
}

\author{
Amani Saad \\ Department of Computer Science, \\ Alexandria University, \\ Alexandria 21544, Egypt
}

\begin{abstract}
In some key database applications, such as data mining, a sequence of interdependent queries may be posed simultaneously to the DBMS. The optimization of such sequences is called multi-query optimization, and it attempts to exploit these dependencies in the derivation of a query evaluation plan (qep). Although it has been observed and demonstrated by several researchers that exploitation of dependencies speed up the query processing, limited research has been reported how to benefit from multi-query optimization, taking the capabilities of existing query optimizers into account. This is exactly the topic of this paper. Since existing optimizers are able to optimize queries in which a restricted number of basic operations appears, e.g., number of joins is limited to 10 , and the optimization of a query is relatively expensive, we attempt to profit from multi query optimization under the condition that queries are passed only once and separately to the optimizer. We propose a two-step optimization procedure. In the first step, we determine, on the basis of the dependencies between queries, in which order they should be specified and what results should be stored. In the second step, each query is passed separately to an optimizer.
\end{abstract}

CR Subject Classification(1991: Database systems (H.2.4) query processing.

Keywords and Phrases: multi-query optimization, architectures, exploiting interdependencies between queries. 


\section{INTRODUCTION}

Query optimization has been recognized as an important area in the field of database technology [Ullm89], especially since the introduction of relational systems. Relational systems offer the user to access data via high-level query languages, and it is the responsibility of the system to select efficient plans to process queries, called query evaluation plans (qeps). A qep describes in which order basic operations, such as selections, projections, joins, etc., should be evaluated to obtain the query answer. Much research has been devoted to select automatically efficient qeps [Grae93]. Since the first and most important database applications were produced in administrative areas, research on query optimization was primarily focussed to meet their performance requirements. An assumption often implicitly made is that these applications give mainly rise to independent queries with a limited number of basic operations. This make it possible to select efficient qeps by a complete enumeration or by applying a few effective heuristics. For example, the number of joins involved is generally less than 10 for those applications. Nowadays, optimizers are able to support the performance requirements yielding from administrative areas very well.

As the variety of database applications grows rapidly, its impact on the performance requirements and the pattern of queries passed to the optimizer poses new research challenges. In database applications, such as data mining and decision support systems, a sequence of interdependent queries are passed simultaneously for processing [ChSi96]. Often, complex queries are split into a number of simpler queries whose results are used by the application to derive the desired result. The simpler queries are passed simultaneously to the DBMS for processing. Optimizing such interdependent queries separately leads to performance that is far from optimal. This has led to several approaches to exploit the dependencies between queries such as illustrated by [AlRa94, Jark84, Kim84, LaYa85, Rous82a, Rous82b, Sell88].

In [Jark84], the author describes how common subexpressions can be detected, and used according to their type (e.g., joins, selections, etc.). In [LaYa85], necessary and sufficient conditions are discussed to compute query results from previously executed queries. In [Rous82a, Rous82b], a framework is provided to derive a common query graph from individual query graphs belonging to individual views, in an attempt to speed up view processing. In the common query graph, different ways are presented to produce the result of a view. Then, the effect of indices on the common query graph is studied, and a set of indices is selected. In [Kim84], a two-step optimization is proposed. In the first step, an analysis of database and query characteristics is performed, and a grouping of queries for simultaneous processing is determined. In the second step, each group of queries is processed in the order determined at the first step and intermediate results are stored on disk. In [Sell88], two algorithms are described for multi-query processing. In the first algorithm, an optimal access plan is generated for each query. Then, a global access plan is obtained by merging the optimal access plans of each query, taking common subexpressions into account. In the second algorithm, a number of access plans for each query is considered. Then, on the basis of heuristics an access plan is chosen for each query such that all common subexpressions found among the queries are used effectively. In [AlRa94], it has been demonstrated that rewriting a set of related expressions in the context of each other, such that no resulting common subexpression is weaker than any of the related expressions, is superior than rewriting techniques that induce common subexpressions that are weaker than the set of related expressions. 
In this paper we address the following problem: how to restructure a sequence of queries such that it can efficiently be processed using the optimizing techniques available in the query optimizer of existing DBMSs. The idea of our approach is to determine an order in which a sequence of (sub)queries should be processed, such that we may profit from the dependencies between queries in processing them. Then, each query is passed separately to the optimizer, and the optimizer selects an efficient query evaluation plan.

What distinguishes our approach to optimization of interdependent queries from the beforementioned efforts is that we use an existing optimizer, and view to it as a 'black-box'. This approach avoids re-development of a complex query optimizer and is adaptive to emerging techniques for query optimization. However, one should be aware of the following limitations of using an existing optimizer. First, as noted already, optimizers are able to handle queries efficiently with a limited number of basic operations only. Approaches based on the integration of queries into a single query graph, such as in [AlRa94, Jark84, Rous82a, Rous82b], are not suitable when using existing optimizers. Passing large query graphs would burden an optimizer with an infeasible task. Second, the optimization of a query is a time consuming task [Choe95, FiST88]. Approaches based on many invocations of an optimizer for a single query, such as in [Sell88], will considerably slow down the optimization process.

The remainder of this paper is organized as follows. In Section 2, we discuss 4 possible architectures to integrate techniques that exploit dependencies between queries and conventional optimizers. In Section 3, we discuss model how to reuse existing output of queries in processing new queries. In Section 4, we elaborate our approach, and, in Section 5, we introduce an algorithm based on this approach. The effectiveness of our approach is shown by a realistic case study in Section 6 . Finally, Section 7 concludes the paper.

\section{ArChiteCtURES For MUlti-QUERY optimization}

In this section, we discuss a number of architectures to integrate techniques that exploit dependencies between queries and conventional optimizing techniques. For each architecture, we point out the strong points and flaws. In Figure 1, we have depicted four possible architectures. We note that variants of architectures $1(\mathrm{a})$ and 1(b) have been introduced in [Sell88].

In Figure 1(a), a multi-set of queries arrives at the optimizer. The optimizer selects an efficient global evaluation plan, which contains the processing strategy for all queries. Then, the plan will be executed. In this architecture, conventional optimization techniques and optimization techniques based on dependencies between queries are strongly integrated. We note that the rule base or cost model used by optimizer will become more complex, since the effects that dependencies between queries will have on an evaluation plan should be modelled as well. Such an architecture is suitable for the development of an optimizer from scratch.

In Figure 1(b), a conventional optimizer selects for each query an efficient qep. Then, all qeps are passed to a reuse module, which attempts to profit from the common parts (caused by the dependencies) by computing them only once and to reuse them in qeps. Consequently, query evaluation plans are rewritten before being executed. Query evaluation plans are based on the available storage structures and access structures in a database management system. A qep produced by a Ingres optimizer may differ from a plan produced by Oracle optimizer. 


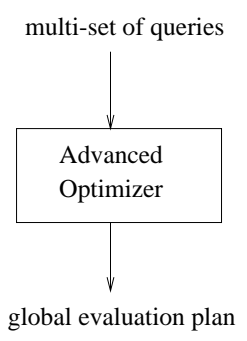

(a)

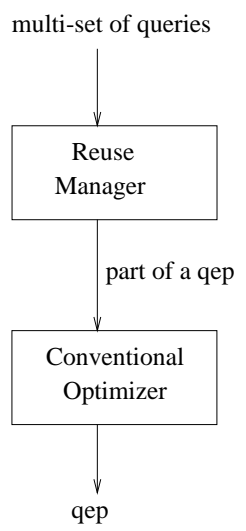

(c)

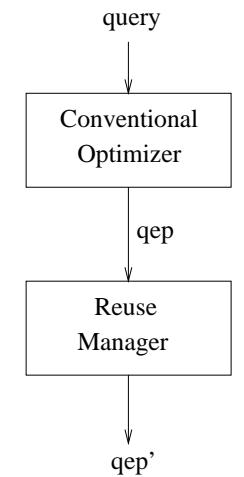

(b)

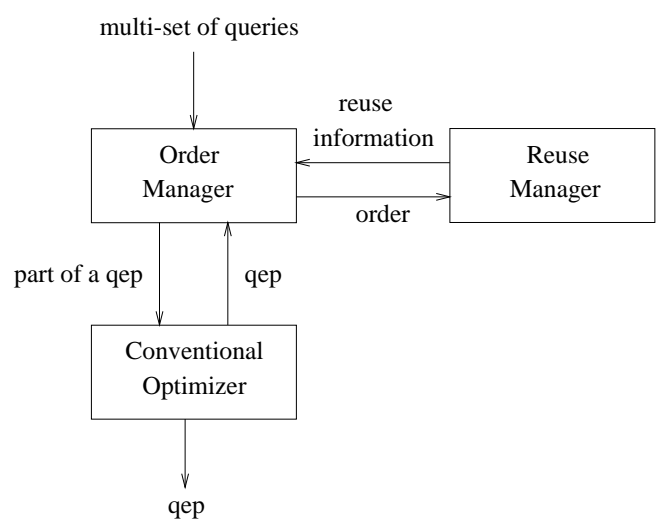

(d)

Figure 1: Architectures

Since the reuse manager attempts to optimize a number of plans by reusing the results of common parts, it is not interested in all details provided by a specific optimizer, such as how a relation is accessed. So, it abstracts from the details and focus on the information relevant for the reuse of earlier computed results, as illustrated in [Sell88]. Once the reuse parts are determined, the abstracted evaluation plan should be augmented with the processing details, in order to be executed by the database system. In fact, architecture 1(b) requires another interface between the optimizer and reuse manager for each kind of database system. Another disadvantage of this architecture is that an optimal plan generated by the conventional optimizer for a query may be killed by the re-use manager, making the effort of the conventional optimizer wasteful.

In Figure 1(c), a multi-set of queries arrives at the reuse manager. The reuse manager determines in which order the queries are to be passed to the conventional optimizer and how to reuse earlier computed results. In fact, the reuse manager determines a part of the qep. An advantage of this architecture above architecture 1(b) is that in principle the same reuse manager can be used for each kind of database system. Furthermore, since the reuse manager determines a part of the qep, it relieves the task of a conventional optimizer. 
In Figure 1(d), the order manager receives a multi-set of queries and chooses an order of execution. Then, it is passed to a reuse module to determine the best way to reuse earlier computed results given this order. After receiving the requested information, it passes each query with the information how to reuse earlier computed results to the optimizer. The optimizer passes on its turn a query evaluation plan to the order module. On the basis of the evaluation plans, the order module may choose another order and the whole procedure may be repeated. This architecture is comparable with architecture 1(c). The difference with architecture 1(c) is that the task of determining an order in which queries should be executed and what and how to reuse earlier computed results are made explicitly in this architecture.

In the remainder of this paper, we will elaborate and implement architecture 1(c) for the following reasons. Commercial database management systems can efficiently handle queries in which a limited number of basic operations appears, e.g., no more than 10 joins [Swam89]. For example, the optimization algorithm used in System R [SACL79] becomes infeasible if the number of joins is larger than 10 [Swam89]. Since we attempt to use existing optimizers in the optimization of interdependent queries, we avoid large query graphs. Therefore, we reject architecture $1(\mathrm{a})$. Since query optimization is a time consuming process, we attempt to limit the number of invocations of an optimizer. Consequently, architecture 1(d) is rejected as well. Finally, we choose architecture 1(c) above 1(b) for the reasons discussed above, namely, the same reuse manager can be used for each kind of database system and it relieves the task of a conventional optimizer.

\section{QUERY PROCESSING}

This section is devoted to a model to store output of queries and how to use them in query processing. Before presenting this model, we briefly outline some preliminaries in Section 3.1.

\subsection{Preliminaries \& assumptions}

In this paper, we deal with relational databases. Each relation $R$ is defined over some attributes, such as, $\alpha_{1}, \alpha_{2}, \ldots, \alpha_{n}$, and is a subset of the Cartesian product $\operatorname{dom}\left(\alpha_{1}\right) \times \operatorname{dom}\left(\alpha_{2}\right) \times$ $\ldots \times \operatorname{dom}\left(\alpha_{n}\right)$, in which $\operatorname{dom}\left(\alpha_{j}\right)$ is the set of values assumed by the $\alpha_{j}$ attributes. For the timebeing, we consider a restricted class of conjunctive queries, i.e., a conjunction of selections and equi-joins in a WHERE clause. A selection is a predicate of the form $R$. $\alpha_{i}$ op constant, in which $o p \in\{=, \neq,<, \leq,>, \geq\}$. An equi-join is a predicate of the form $R_{1} \cdot \alpha_{i}=R_{2} \cdot \alpha_{j}$. Furthermore, we do not consider projections to simplify our analysis without invalidation of the obtained results. Incorporation of projections into our approach is straightforward. Observe that a disjunction of predicates in a WHERE clause of a query can be replaced by a number of queries, for which the WHERE clause consists of a conjunction of predicates. Although we restrict ourselves to a special class of queries, this class contains the most common types of queries.

We assume that a database resides on disk. The main cost factor in this type of databases is still disk accesses [ElNa89]. Therefore, we take disk accesses as cost unit. Furthermore, we assume that a relation is stored as a heap, and no indices are allocated to attributes. We note that a data warehouse, which generally maintains historical information, is a typical application that is implemented in this way. A data warehouse tends to be very large and to grow over time. 


\subsection{Model}

Our approach for multi-query optimization exploits the dependencies between the queries in a sequence. To speed-up query processing, (intermediate) results of queries are temporarily stored and reused. Although our approach can be targeted to different models that store intermediate results, we describe a model to store and reuse intermediate results for illustrative purposes.

We assume that each tuple in a relation has a unique tuple identifier (tid). Instead of storing tuples that qualify as intermediate results, we store its tid in main memory. We assume that a main memory is large enough to store all tids that qualify as intermediate results. So, an intermediate results can be regarded as a relation $T$, called tid-relation, in which attribute $\alpha_{i}$.tid assumes the tid values of relation $R_{i}$. For example, storage of intermediate results due to a join between relations $R_{i}$ and $R_{j}$ leads to the storage of a tid-relation $T_{i, j}$ with two attributes, one assuming the tid values of $R_{i}$ and the other assuming tid values of $R_{j}$, in main memory. A consequence of this storage model for intermediate results is that whenever one needs a tuple, this should be retrieved from disk. Before illustrating how to use tidrelations in query processing, we present a definition for the intersection of two tid-relations that resides in main memory.

Definition 3.1: Let $T_{R_{1}, R_{2}, R_{3} \ldots, R_{n}}=\left(\alpha_{1}, \alpha_{2}, \alpha_{3}, \ldots, \alpha_{n}\right)$ and $T_{S_{1}, S_{2}, S_{3}, \ldots, S_{m}}=$ $\left(\beta_{1}, \beta_{2}, \beta_{3}, \ldots, \beta_{m}\right)$ be relations, in which $\operatorname{dom}\left(\alpha_{i}\right), i \leq n$, and $\operatorname{dom}\left(\beta_{i}\right), j \leq m$, is the set of tid values that appears in relation $R_{i}$ and $S_{j}$, respectively. Let $u . \alpha$ represents the value of an attribute $\alpha$ in a tuple $u$. Then, the intersection of $T_{R_{1}, R_{2}, R_{3} \ldots, R_{n}}$ and $T_{S_{1}, S_{2}, S_{3}, \ldots, S_{m}}$ is

$$
\begin{aligned}
\cap\left(T_{R_{1}, R_{2}, R_{3} \ldots, R_{n}}, T_{S_{1}, S_{2}, S_{3}, \ldots, S_{m}}\right)= & \left\{u v \mid u \in T_{R_{1}, R_{2}, R_{3} \ldots, R_{n}} ; v \in T_{S_{1}, S_{2}, S_{3}, \ldots, S_{m}} ;\right. \\
& \left.\forall \alpha_{i}, \beta_{j}: \operatorname{dom}\left(\alpha_{i}\right)=\operatorname{dom}\left(\beta_{j}\right) \Rightarrow u . \alpha_{i}=v . \beta_{j}\right\}
\end{aligned}
$$

Let us continue by illustrating how to use tid-relations in query processing by means of an example.

Example 3.1: Consider the relational schema and queries defined in Figure 2 with the following content.

Emp
\begin{tabular}{|c|c|c|c|c|}
\hline tid & name & age & salary & dept-name \\
\hline 601 & Tutiram & 30 & $60 \mathrm{~K}$ & AA \\
603 & Tataram & 34 & $40 \mathrm{~K}$ & AA \\
609 & Totaram & 22 & $8 \mathrm{~K}$ & AP \\
610 & Titaram & 26 & $15 \mathrm{~K}$ & AP \\
621 & Jansen & 40 & $30 \mathrm{~K}$ & AP \\
623 & Pincho & 31 & $9 \mathrm{~K}$ & IS \\
624 & Oeroeg & 45 & $20 \mathrm{~K}$ & AA \\
$\ldots$ & $\ldots .$. & $\ldots$ & $\ldots$ & $\ldots$ \\
\hline
\end{tabular}

Dept
\begin{tabular}{|c|c|c|}
\hline tid & dept-name & num-of-emps \\
\hline 402 & AA & 14 \\
404 & AP & 32 \\
407 & IS & 8 \\
\hline
\end{tabular}

The queries $q_{1}, q_{2}$, and $q_{3}$ will be processed according to the following plan. First, query $q_{1}$ is resolved. Then, the following intermediate query, $q_{i n t}$, is resolved. The tids of tuples that satisfy to these queries will be stored in main memory. 
Relations:

Emp(name, age, salary, dept-name)

Dept(dept-name, num-of-emps)

Queries:

$q_{1}:$ SELECT * FROM Emp, Dept

WHERE Emp.dept-name $=$ Dept.dept-name

AND Emp.age $\leq 40$

AND Dept.num-of-emps $\leq 20$

$q_{2}:$ SELECT * FROM Emp, Dept

WHERE Emp.dept-name $=$ Dept.dept-name

AND Emp.age $\leq 50$

AND Dept.num-of-emps $\leq 10$

$q_{3}:$ SELECT $*$ FROM Emp, Dept

WHERE Emp.dept-name $=$ Dept.dept-name

AND Emp.age $\leq 40$

AND Dept.num-of-emps $\leq 15$

AND Emp.salary $\geq 10.000$

Figure 2: Relational schema and queries defined on schema

$q_{\text {int }}:$ SELECT * FROM Emp, Dept

WHERE Emp.dept-name $=$ Dept.dept-name

AND Emp.age $\leq 50$

AND Dept.num-of-emps $\leq 15$

Finally, $q_{3}$ is resolved by using the results of the intermediate query $q_{i n t}$ and $q_{1}$, and $q_{2}$ by using $q_{\text {int }}$.

The following relations will be stored in main memory due to the results of $q_{1}$ and $q_{\text {int }}$.

$\operatorname{res}\left(\mathrm{q}_{1}\right)$
\begin{tabular}{|l|c|}
\hline E.tid & D.tid \\
\hline 601 & 402 \\
603 & 402 \\
623 & 407 \\
\hline
\end{tabular}

$\operatorname{res}\left(\mathrm{q}_{\text {int }}\right)$
\begin{tabular}{|c|c|}
\hline E.tid & D.tid \\
\hline 601 & 402 \\
603 & 402 \\
623 & 407 \\
624 & 402 \\
\hline
\end{tabular}

Using the result of $q_{\text {int }}$ that is stored in main memory, $q_{2}$ may be processed as follows. For each tuple $t$ in $q_{i n t}$, tuple $u$ in relation Dept, whose tid corresponds to the D.tid value of $t$, is retrieved. If this tuple satisfies to the restriction on num-of-emps $(\leq 10)$, tuple $v$ in $E m p$, whose tid corresponds to the E.tid value of $t$, is retrieved. Then, tuples $u$ and $v$ are concatenated.

Query $q_{3}$ is processed as follows. The intersection between $\operatorname{res}\left(q_{1}\right)$ and res $\left(q_{\text {int }}\right)$ is computed, which results into res $\left(q_{1}\right)$. So, this means that res $\left(q_{1}\right)$ contains all tids of tuples that satisfies to $q_{3}$, except for the restriction on Emp.salary. To output the result that satisfies also to this restriction, a similar procedure can be used as in the processing of $q_{2}$. 


\begin{tabular}{|c|c|c|}
\cline { 2 - 3 } \multicolumn{1}{c|}{} & $q_{2}$ & $q_{3}$ \\
\hline$q_{1}$ & Emp.dept-name $=$ Dept.dept-name & $q_{1}$ \\
& Emp.age $\leq 50$ & \\
& Dept.num-of-emps $\leq 20$ & Emp.dept-name $=$ Dept.dept-name \\
& - & Emp.age $\leq 50$ \\
& & Dept.num-of-emps $\leq 15$ \\
\hline
\end{tabular}

Figure 3: csq matrix corresponding to Figure 2

From the example it should be clear that the cost entailed by using tid relations in processing queries depends on the different number of tuples that should be retrieved from disk from each relation. Once, this is known the cost involved in retrieving $t$ tuples from $m$ pages containing $n(>m)$ tuples can be estimate by the well-known formula presented in [Yao77]. For rough estimation of the number of tuples that satisfies to a selection or join, we refer to [Ullm89]. Given the formulae for these estimations, the derivation of a rough cost model for above-mentioned query processing technique is straightforward.

\section{EXPLOITING INTERDEPENDENCIES BETWEEN QUERIES}

In this section, we study how to re-structure a sequence of queries such that it can be efficiently processed by an optimizer. Re-structuring a sequence of queries means that a new sequence of queries is determined and the order in which these queries should be passed to the optimizer is established. A query in the new sequence is either a query coming from the original sequence or is an intermediate query, which is derived to speed-up a number of queries in the original sequence. Such an intermediate query is called a common subquery. In Section 4.1, we precisely define what is meant by a common subquery. Then, in Section 4.2 , we exploit common subqueries in our approach.

\subsection{Common subquery}

Our approach is based on the exploitation of results of common subqueries between two queries. The result of a common subquery (csq) of two queries $q_{i}$ and $q_{j}$ is a set of tuples that contains the result of both $q_{i}$ and $q_{j}$. For example, a common subquery for queries $q_{2}$ and $q_{3}$ in Figure 2 is the query $q_{i n t}$ in Example 3.1. In the following, we formalize the notion of common subquery.

Definition 4.1: A selection $s_{i}$ subsumes a selection $s_{j}, s_{i} \Rightarrow s_{j}$, if $s_{i}$ and $s_{j}$ are defined over the same relational schema and the set of tuples satisfying $s_{i}$ is a subset of those tuples satisfying $s_{j}$. Selections $s_{i}$ and $s_{j}$ are equal, $s_{i}=s_{j}$, iff $s_{i} \Rightarrow s_{j}$ and $s_{j} \Rightarrow s_{i}$.

Definition 4.2: Let $\mathcal{S}_{i}$ represent the set of selections and $\mathcal{E}_{i}$ the set of equi-joins in the WHERE clause of a query $q_{i}$. A query $q_{i, j}$ is a common subquery of queries $q_{i}$ and $q_{j}$, in which $i \neq j$, if $\mathcal{S}_{i, j}$ contains all selections $s_{i, j}$ for which holds: $\exists s_{i} \in \mathcal{S}_{i} ; \exists s_{j} \in$ $\mathcal{S}_{j} ;\left(s_{i} \Rightarrow s_{i, j} \wedge s_{j}=s_{i, j}\right) \vee\left(s_{i}=s_{i, j} \wedge s_{j} \Rightarrow s_{i, j}\right)$ and $\mathcal{E}_{i, j}$ contains all equi-joins $e_{i, j}$ for which holds: $e_{i, j} \in \mathcal{E}_{i} \wedge e_{i, j} \in \mathcal{E}_{j}$ 


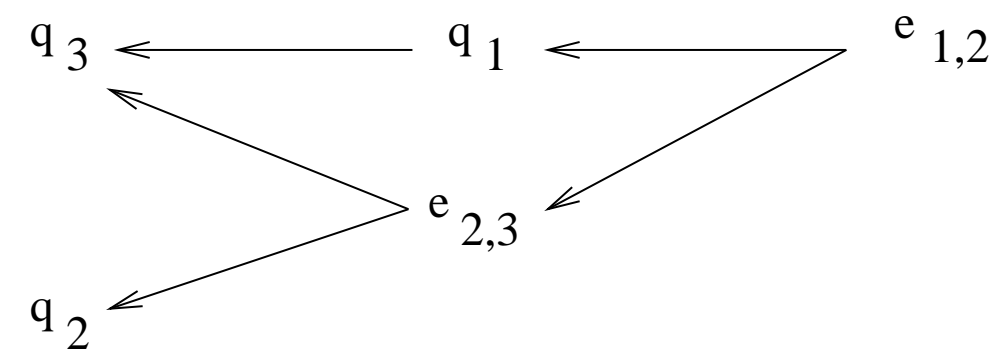

Figure 4: Relationship graph corresponding to Figure 2

The detection of common subqueries is beyond the scope of this paper. Several algorithms have been proposed to detect common subqueries [Fink82, RoHu80]. In the remainder of this paper, we assume that a common subquery can be generated.

\subsection{Approach}

Before presenting our approach, we introduce the notion of a common subquery matrix, abbreviated as csq-matrix. A csq-matrix for a sequence of $n$ queries has size of $(n-1)$ by $(n-1)$. An element $e_{i, j}, i<j$, represents the WHERE clause of the common subquery of queries $q_{i}$ and $q_{j}$. Since elements $e_{i, j}$ and $e_{j, i}, i \neq j$ concern the same WHERE clause, we omit the clause for $e_{j, i}$. Furthermore, the value of an element $e_{i, i}$ is not defined, since a common subquery with regard to a single query is not defined. An example of a csq-matrix, which regard to the relational schema and queries of Figure 2, is given in Figure 3. The first element $e_{1,2}$ contains the WHERE clause of the subquery with regard to the queries $q_{1}$ and $q_{2}$. We note that if the common subquery of two queries $q_{i}$ and $q_{j}$ is $q_{i}$, then we denote, for convenience's sake, $q_{i}$ in a csq-matrix and not its WHERE clause. For example, from Figure 3 , we see that $q_{1}$ and $q_{3}$ have $q_{1}$ as common subquery.

Our approach to optimize a sequence of interdependent queries consists of two phases. In the first phase, we derive from the csq-matrix the set of common subqueries that may be used in computing the output of a query $q$. We apply some rules to limit the elements in this set. Then, we build up a graph that establishes the relationships between the output of all remaining (common sub)queries. The graph corresponding to the queries of Figure 2 and its csq-matrix is given in Figure 4. An edge from a node $n_{i}$ to a node $n_{j}$ means that the output of the query corresponding to $n_{i}$ contains the output of the query corresponding to $n_{j}$. Therefore, the output of $n_{i}$ can be used in computing the query corresponding to $n_{j}$.

In the second phase, we analyse the nodes that correspond to a query that does not belong to the initial sequence of queries, called intermediate nodes. In Figure $4, e_{1,2}$ and $e_{2,3}$ are intermediate nodes.

If the output of a query corresponding to an intermediate node can be obtained by an intersection of the (available) output of other nodes, this intermediate node is kept into the graph. The reason is that there is no need to retrieve tuples from base relations in this case and intersections can be cheaply performed. Whenever it happens that the output of such a query will not be used in the computation of other queries, the loss of efficiency is limited. In all other cases, we estimate the investments in computing queries corresponding 
to intermediate nodes and the return on investments. On the basis of these estimations, it is decided whether an intermediate node will be discarded or not. For example, in Figure 4, node $e_{1,2}$ will be deleted if we expect that the sum of the cost to compute $q_{1}$ and $q_{2}$ without using $e_{1,2}$ is less than using $e_{1,2}$. Similarly, $e_{2,3}$ will be deleted if the sum of the cost to compute $q_{2}$ and $q_{3}$ without using $e_{2,3}$ is less than using $e_{2,3}$. Of course, the cost to compute the output of a query corresponding to an intermediate node should be taken into account in the decision whether a node should be discarded or not. We note that the cost to compute the query corresponding to $e_{2,3}$ depends on whether $e_{1,2}$ is discarded or not.

In two consecutive subsections, we discuss the phases of our approach, and the rationales behind them in more detail.

4.2.1 Phase 1 From a csq-matrix we can derive all common subqueries to evaluate a query $q_{i}$ (and at least one other query) from a sequence of queries. Consider a csq-matrix with regard to a sequence of queries, $S=q_{1}, q_{2}, q_{3}, \ldots, q_{n}$. Let $\mathcal{Q}_{j}^{<}=\cup_{i<j} e_{i, j}$ and $\mathcal{Q}_{j}^{>}=\cup_{j<i \leq n} e_{j, i}$, in which $e_{i, j}$ is an element of the csq-matrix and $e_{i, j} \neq q_{j}$. Then, $\mathcal{Q}_{j}=\mathcal{Q}_{j}^{<} \cup \mathcal{Q}_{j}^{>}$contains all the queries whose output can be used in processing query $q_{j}$. For example, query $q_{1}, q_{2}$ and $q_{3}$ in Figure 2 can be evaluated using a subset of $\mathcal{Q}_{1}=\left\{e_{1,2}\right\}, \mathcal{Q}_{2}=\left\{e_{1,2}, e_{2,3}\right\}$, and $\mathcal{Q}_{3}=\left\{q_{1}, e_{2,3}\right\}$, respectively, in which $e_{i, j}$ is the i-jth element of the csq-matrix of Figure 3. To limit the number of elements of $\mathcal{Q}_{j}$, we present the following two rules.

Rule 1: Let $q_{i}$ and $q_{j}$ belong to a sequence of queries $S$, and $\mathcal{Q}_{j}$ contains the set of common subqueries whose output can be used to process query $q_{j}$. If $q_{i}$ and $q_{k}$ are elements of $\mathcal{Q}_{j}$ and the output of $q_{i}$ is a subset of the output of $q_{k}$, thus output $\left(q_{i}\right) \subseteq \operatorname{output}\left(q_{k}\right)$, then $q_{k}$ can be deleted from $\mathcal{Q}_{j}$.

The rationale behind this rule is based on the following. Since query $q_{i}$ belongs to sequence $S$, it should be computed. The computation of the output of $q_{j}$ from a smaller amount of tuples is cheaper than from a larger amount, and, therefore, it is better to use $q_{i}$ instead of $q_{k}$. We note that, although this argument holds even if $q_{i}$ does not belong to $S$, Rule 1 is hard to defend in this case, because we will suffer from information loss. If $q_{i}$ does not belong to $S$, it is not certain that $q_{i}$ will be evaluated; this depends on the gain that we expect from using the output of $q_{i}$ in evaluating other queries. Suppose it is not profitable to evaluate $q_{i}$ and we have discarded $q_{k}$ from $\mathcal{Q}_{j}$, then we have lost the information that $q_{k}$ can be used for the evaluation $q_{k}$.

The second rule to reduce the size of a graph looks as follows.

Rule 2: Let $q_{i}, q_{k}, q_{k+1}, \ldots, q_{m} \in \mathcal{Q}_{j}$, and $q_{j}, q_{k}, q_{k+1}, \ldots, q_{m}$ belong to a sequence $S$, while $q_{i}$ does not belong to $S$. If output $\left(q_{i}\right)=\operatorname{output}\left(q_{k}\right) \cap \operatorname{output}\left(q_{k+1}\right) \cap \ldots \cap \operatorname{output}\left(q_{m}\right)$, then $q_{i}$ can be discarded from $\mathcal{Q}_{j} \square$.

The rationale behind this rule is as follows. Let it be possible that the output of a common subquery $q_{s}$ that does not belong to $S$ can be obtained by the intersection of output of queries belonging to $S$. Then, such a query does not require retrievals from base relations. Since queries belonging to $S$ should be computed anyway, the output of $q_{s}$ can be obtained for some 
$\operatorname{lqp}\left(e_{1,2}\right)=\bowtie_{\text {dept-name }}=\operatorname{dept-name}\left(\left(\sigma_{\text {age } \leq 50}(E m p)\right),\left(\sigma_{\text {num-of-emps } \leq 20}(D e p t)\right)\right)$

$\operatorname{lqp}\left(q_{1}\right)=\sigma_{\text {age } \leq 40}\left(\operatorname{output}\left(e_{1,2}\right)\right)$

$\operatorname{lqp}\left(q_{2}\right)=\sigma_{\text {num-of-emps } \leq 20}\left(\right.$ output $\left.\left(e_{2,3}\right)\right)$

$\operatorname{lqp}\left(q_{3}\right)=\sigma_{\text {salary } \geq 10.000}\left(\cap\left(\right.\right.$ output $\left(e_{2,3}\right)$, output $\left.\left.\left(q_{1}\right)\right)\right)$

Figure 5: Examples of logical query plans

CPU cost. Therefore, there is no need to consider $q_{s}$ for further analysis. We note that, in general, computing $q_{s}$ from scratch will be more expensive than computing it from available output of queries.

In Section 6, we demonstrate the effectiveness of above-mentioned rules by means of a case study.

4.2.2 Phase 2 Phase 2 takes as input a graph produced in phase 1. To minimize the processing cost of a sequence $S$, we analyse whether the output of the corresponding query of a node contributes to this goal or not. We divide the nodes of a graph into two groups.

1. The first group contains nodes that correspond to queries that appear in $S$.

2. The second group contains all other nodes, i.e., nodes that correspond to queries that do not appear in $S$ and require joins with base relations or selections on base relations to compute their output.

In phase 2, we focus on the analyse of queries corresponding to nodes of group 2, i.e., we estimate the investment to compute the output of a node and the return on investment. Since the output of the queries of group 1 should be computed anyway, they are not analysed.

The analysis of queries of group 2 will be done on the basis of cost estimations. Before introducing our analysing technique, we introduce the notion of logical query plan (lqp). A logical query plan is a sequence of operations on a number of sets of tuples. The operations in a lqp are selection $(\sigma)$ on relations ${ }^{1}$, join $(\bowtie)$ between relations, and the intersection $(\cap)$ of tid-relations. A lqp of a query $q$ represents how the output of $q$ may be computed by making use of available output of queries and base relations. To generate lqps, we use the following intuitively appealing heuristics [Ullm89]. We perform first the intersection on tuple identifiers of relations that are stored in main memory, then selections, and finally joins. Furthermore, all output of queries in main memory that can be used, and are available, will be used. Figure 5 represents a logical query plan for the queries corresponding to the nodes $e_{1,2}, q_{1}, q_{2}$, and $q_{3}$ of the graph in Figure 4.

In order to analyse a query corresponding to an intermediate node, let say $e_{k, l}$, in which joins are involved, we distinguish two sets of queries. The first set, $S Q 1_{k, l}$, consists of queries that appear in the initial sequence $S$ and which may be computed by a selection on the output of the query corresponding to $e_{k, l}$ or by a selection on a subset of the output of the query corresponding to $e_{k, l}$. In the following formal descriptions of the sets, we mean by a

\footnotetext{
${ }^{1}$ We note that the output of a query is a derived relation.
} 
node $e_{k, l}$ its corresponding query.

$$
\begin{aligned}
S Q 1_{k, l}= & \left\{q \mid q \in S \wedge\left(\left(\operatorname{lqp}(q)=\sigma_{\alpha \text { op constant }}\left(\text { output }\left(e_{k, l}\right)\right)\right) \cup(\operatorname{lqp}(q)=\right.\right. \\
& \left.\left.\left.\sigma_{\alpha \text { op constant }}\left(\text { output }\left(e_{p, q}\right)\right) \wedge e_{p, q} \notin S \wedge e_{k, l} \text { is the csq of } e_{k, l} \text { and } e_{p, q}\right)\right)\right\}
\end{aligned}
$$

The first part of our rule to evaluate nodes in phase 2 is based on $S Q 1_{k, l}$. Let $\operatorname{Cost}\left(S Q 1_{k, l}\right)$ be the sum of the processing cost of the queries of $S Q 1_{k, l}$ using the output of the query corresponding to $e_{k, l}$, while $\operatorname{Cost}(S Q 1)$ represents the cost not using this output. Then, Rule 3a looks as follows:

Rule 3a: Let $G$ be a graph and $e_{k, l}$ a node. If $\operatorname{Cost}\left(S Q 1_{k, l}\right)+\operatorname{Cost}\left(e_{k, l}\right)<\operatorname{Cost}(S Q 1)$, then $e_{k, l}$ remains in $G$.

The values for Cost(.) may be obtained by passing each query to the optimizer and asking for an estimate, or they can be estimated on the basis of a rough cost model.

For the second part of Rule 3, we introduce the set $S Q 2_{k, l}$. This set considers queries with corresponding lqp, in which the output of $e_{k, l}$ is involved, but is not simply a selection on the output of $e_{k, l}$, and the removal of the output of $e_{k, l}$ will lead to a lqp in which more joins will be involved than in the initial lqp. Let $J_{l q p}$ represent the number of joins involved in a logical query plan lqp, and let the operation remove $\left(l q p\right.$, output $\left.\left(e_{k, l}\right)\right)$ return a logical query plan lqp', such that lqp' does not contain the output of $e_{k, l}$. Then $S Q \mathscr{2}_{k, l}$ is defined as

$$
\begin{aligned}
S Q \mathscr{2}_{k, l}= & \left\{q \mid q \in S \wedge q \notin S Q 1 \wedge \text { output }\left(e_{k, l}\right) \in l q p(q) \wedge\right. \\
& \text { remove } \left.\left(\operatorname{lqp}, \text { output }\left(e_{k, l}\right)\right) \Rightarrow J_{l q p^{\prime}}>J_{l q p}\right\}
\end{aligned}
$$

This set will be used in Rule $3 \mathrm{~b}$. We note that this rule is applied on nodes for which no statement could be made by Rule 3 a.

Rule 3b: Let $G$ be a graph, $e_{k, l}$ a node, and $\operatorname{Cost}\left(S Q 1_{k, l}\right)+\operatorname{Cost}\left(e_{k, l}\right) \geq \operatorname{Cost}(S Q 1)$. If $\operatorname{Cost}\left(S Q 1_{k, l}\right)+\operatorname{Cost}\left(S Q 2_{k, l}\right)+\operatorname{Cost}\left(e_{k, l}\right)<\operatorname{Cost}(S Q 1)+\operatorname{Cost}(S Q 2)$ then $e_{k, l}$ remains in $G$, else $e_{k, l}$ is discarded.

In the next section, we present an algorithm to implement the approach discussed so far.

\section{An ALGORITHM}

The algorithm takes as input a sequence of interdependent queries, $S$, and produces a list of queries, $L$. The number of queries in $L$ is larger or equal to the number of queries in $S$. It should be clear that additional queries to $L$ are added to speed up the evaluations of other queries. The body of the algorithm consists of the following 4 steps. We discuss each of these steps.

1. In the first step a csq-matrix is build with regard to the queries of $S$. For each common subquery $q_{\not S}$, we check whether $q_{\not S}$ is equal to a query $q_{S}$ that belong to the sequence $S$. If this is the case $q_{\not \supset}$ is replaced by $q_{S}$. Finally, we derive for each query, $q$, the set containing all queries whose output can be used in computing $q, \mathcal{Q}$, as discussed in Section 4.2.1. 


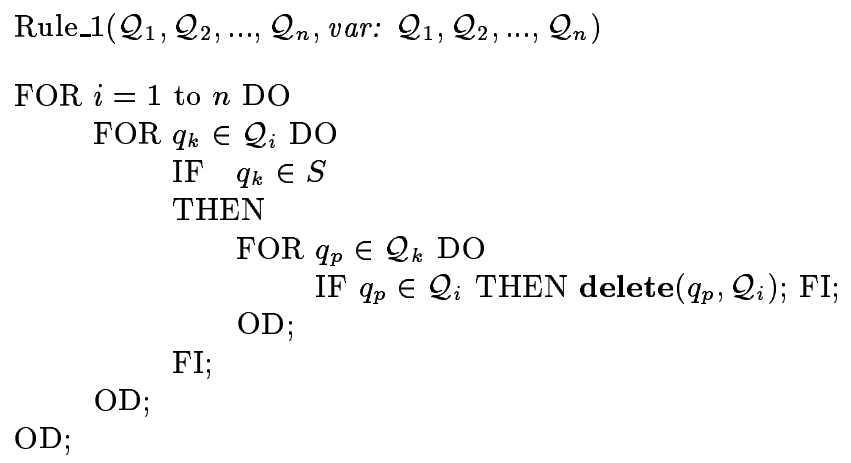

(a)

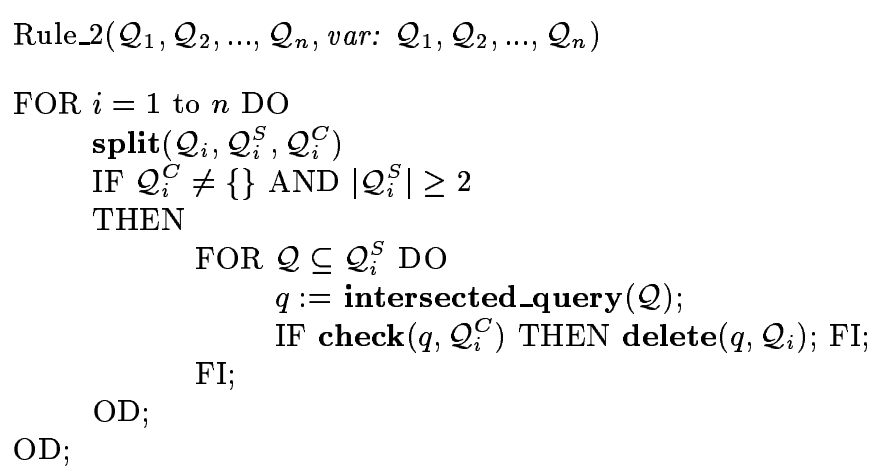

Figure 6: Procedures for Rule 1 and Rule 2

2. Rules 1 and 2 are successively applied on each $\mathcal{Q}_{i}$.

3. Steps 1 and 2 are repeated for common subqueries that do not belong to the initial sequence $S$. This step establishes the relationships between these common subqueries and between these common subqueries and queries belonging to $S$. Then, a graph is built up on the basis of the obtained results so far.

4. Each intermediate node is evaluated according to Rule 3a and Rule 3b.

In the literature, algorithms are described to perform parts of above-mentioned steps. It is not our intention to describe similar algorithms for these parts. In the following, we discuss the implementation of the parts of each step that is not straightforward and for which no algorithms are described in literature.

The core of step 1 is to build a csq matrix with regard to the queries of $S$. We have already noticed that a csq matrix can be generated by using algorithms described in [Fink82, RoHu80]. In Section 4.2.1, we have described how to obtain for each query $q$ its corresponding set $\mathcal{Q}$ from the csq matrix. 
More effort is required for the application of rules 1 and 2 in step 2 . Let us describe algorithms to perform these rules. Rule 1 can be applied as follows. A query $q_{k} \in \mathcal{Q}_{i}$, such that $q_{k} \in S$, is picked. Then, all elements that appear in $\mathcal{Q}_{k}$ can be deleted from $\mathcal{Q}_{i}$, since the output of each query corresponding to an element in $\mathcal{Q}_{k}$ is a superset of the output of $q_{k}$. In Figure 6(a), the pseudo-code is presented.

For the time being, we apply Rule 2 in a naive way. A set $\mathcal{Q}_{i}$ is split into two sets $\mathcal{Q}_{i}^{S}$ and $\mathcal{Q}_{i}^{C}$. Set $\mathcal{Q}_{i}^{S}$ contains the queries of $\mathcal{Q}_{i}$ that also belong to the initial sequence $S$, while $\mathcal{Q}_{i}^{C}$ contains all other queries of $\mathcal{Q}_{i}$. If $\mathcal{Q}_{i}^{C} \neq\{\}$ and $\mathcal{Q}_{i}^{S}$ contains at least two elements, we determine for each subset $\mathcal{Q}_{s u b} \subseteq \mathcal{Q}_{i}^{S}$, the intersection of the queries of $\mathcal{Q}_{\text {sub }}$, called intersected query. Then, we check for each query in $\mathcal{Q}_{i}^{C}$ whether it can be replaced by the intersected query. We note that the output of an intersected query of a set $\mathcal{Q}$ is the greatest common set of tuples with regard to the queries in this set. The WHERE clause of an intersected query $q$ of two queries $q_{j}$ and $q_{k}$ can be obtained by taking the union of the WHERE clauses of $q_{j}$ and $q_{k}$. In Figure 6b, the pseudo-code for Rule 2 is presented.

Since step 3 is a repetition of steps 1 and 2, the implementation of this step is similar to the implementation of steps 1 and 2 .

Finally, step 4 involves the application of Rule 3a and Rule 3b. As described in Section 4.2.2, the application of these rules requires logical query plans. The generation of logical query plans, as described in Section 4.2.2, from SQL is a well-understood subject, and, therefore, it is omitted from this paper. Once logical query plans are available, Rule 3a and Rule $3 \mathrm{~b}$ can be applied as discussed in Section 4.2.2.

\section{A CASE STUDY}

In this section, we illustrate the effectiveness of the rules in our approach by means of a realistic case that has been introduced in [Fink82] and slightly modified and used in [Sell88]. The case consists of 8 queries defined on 3 relations. We have adopted the modified version of this case as presented in [Sell88]. The queries and relations are presented in Figure 7.

In Figure 8(a), the csq matrix is presented with regard to the queries in Figure 7 and in Figure 8 (b) the set of queries, $\mathcal{Q}$, that can be used in processing a query $q$. For a $q_{i}, \mathcal{Q}_{i}$ can be obtained by taking the union of the cells corresponding to row $q_{i}$ and column $q_{i}$ in a csq-matrix. Thus, from Figure 8(a) follows that $\mathcal{Q}_{6}=\left\{\right.$ output $\left(q_{1}\right)$, output $\left(q_{2}\right)$, output $(B)$, output $\left(q_{4}\right)$, output $(C)\} \cup\left\{\right.$ output $\left.\left(q_{8}\right)\right\}$

The application of Rule 1 results into the following sets:

$$
\begin{aligned}
& \mathcal{Q}_{1}=\{\} \\
& \mathcal{Q}_{2}=\left\{\text { output }\left(q_{1}\right), \text { output }(A)\right\} \\
& \mathcal{Q}_{3}=\left\{\text { output }\left(q_{1}\right), \text { output }(B)\right\} \\
& \mathcal{Q}_{4}=\left\{\text { output }\left(q_{1}\right), \text { output }(A), \text { output }(B)\right\} \\
& \mathcal{Q}_{5}=\left\{\text { output }\left(q_{3}\right), \text { output }(C), \text { output }(D), \text { output }\left(q_{8}\right)\right\} \\
& \mathcal{Q}_{6}=\left\{\text { output }(C), \text { output }\left(q_{8}\right)\right\} \\
& \mathcal{Q}_{7}=\left\{\text { output }\left(q_{3}\right), \text { output }(D), \text { output }\left(q_{8}\right)\right\} \\
& \mathcal{Q}_{8}=\left\{\text { output }\left(q_{2}\right), \text { output }\left(q_{4}\right)\right\}
\end{aligned}
$$


Relations:

$E$ (mployee) (name, empl(oyer), age, exp(erience), salary, educ(ation))

$C$ (orporation)(cname, loc(ation), earnings, pres(ident), business)

$S(\mathrm{chool})($ sname, level)

Queries:

$q_{1}:$ SELECT $*$ FROM $E$ WHERE E.exp $\geq 10$

$q_{2}:$ SELECT $*$ FROM $E$ WHERE E.exp $\geq 20$ AND E.age $\leq 65$

$q_{3}:$ SELECT $*$ FROM E, C WHERE E.empl $=$ C.cname AND E.exp $\geq 10$

AND C.earnings $>500$ AND C.loc $\neq$ 'Kansas'

$q_{4}:$ SELECT * FROM E, C WHERE E.empl = C.cname AND E.exp $\geq 20$

AND $\quad$ C.earnings $>300$ AND C.loc $\neq$ 'Kansas'

$q_{5}:$ SELECT $*$ FROM $E, C$ WHERE E.empl $=$ C.cname AND E.empl $=$ C.pres

AND E.exp $\geq 20 \quad$ AND E.age $\leq 65$

AND C.earnings $>500$ AND C.loc $=$ 'New York'

$q_{6}:$ SELECT $*$ FROM $E, C$ WHERE E.empl $=$ C.cname AND E.empl $=$ C.pres

AND E.exp $\geq 30 \quad$ AND E.age $\leq 60$

AND C.earnings $>300$ AND C.loc $=$ 'New York'

$q_{7}:$ SELECT $*$ FROM E, $C, S$ WHERE E.empl = C.cname AND E.educ $=$ S. sname

AND E.exp $\geq 20 \quad$ AND E.age $\leq 65$

AND C.earnings $>500$ AND C.loc $=$ 'New York'

AND $\quad$ S.level $=$ 'univ'

q8: SELECT $^{*}$ FROM E, C WHERE E.empl $=$ C.cname AND E.exp $\geq 20$

AND E.age $\leq 65 \quad$ AND C.earnings $>300$

AND $\quad$ C.loc $=$ 'New York'

Figure 7: Relational schema and workload description 


\begin{tabular}{|c|c|c|c|c|c|c|c|}
\hline & $\mathrm{q}_{2}$ & $\mathrm{q}_{3}$ & $\mathrm{q}_{4}$ & $\mathrm{q}_{5}$ & $\mathrm{q}_{6}$ & $\mathrm{q}_{7}$ & $\mathrm{q}_{8}$ \\
\hline $\mathrm{q}_{1}$ & $\mathrm{q}_{1}$ & $\mathrm{q}_{1}$ & $\mathrm{q}_{1}$ & $\mathrm{q}_{1}$ & $\mathrm{q}_{1}$ & $\mathrm{q}_{1}$ & $\mathrm{q}_{1}$ \\
\hline $\mathrm{q}_{2}$ & - & $\mathrm{q}_{1}$ & E.exp $\geq 20=A$ & $\mathrm{q}_{2}$ & $\mathrm{q}_{2}$ & $\mathrm{q}_{2}$ & $\mathrm{q}_{2}$ \\
\hline $\mathrm{q}_{3}$ & - & - & $\begin{array}{l}\text { E.empl = C.cname } \\
\text { E.exp } \geq 10 \\
\text { C.earnings > } 300 \\
\text { C.loc ₹ 'Kansas' } \\
\quad \| \\
\text { B }\end{array}$ & $\mathrm{q}_{3}$ & $\begin{array}{l}\text { E.empl = C.cname } \\
\text { E.exp } \geq 10 \\
\text { C.earnings > } 300 \\
\text { C.loc ₹ 'Kansas' } \\
\text { ॥ } \\
\text { B }\end{array}$ & $\mathrm{q}_{3}$ & $\begin{array}{l}\text { E.empl }=\text { C.cname } \\
\text { E.exp } \geq 10 \\
\text { C.earnings }>300 \\
\text { C.loc } \neq \text { 'Kansas' } \\
\| \\
\text { B }\end{array}$ \\
\hline $\mathrm{q}_{4}$ & - & - & - & $\mathrm{q}_{4}$ & $\mathrm{q}_{4}$ & $\mathrm{q}_{4}$ & $\mathrm{q}_{4}$ \\
\hline $\mathrm{q}_{5}$ & - & - & - & - & $\begin{array}{l}\text { E.empl }=\text { C.cname } \\
\text { E.emp }=\text { C.pres } \\
\text { E.exp } \geq 20 \\
\text { E.age } \leq 65 \\
\text { C.loc }=\text { 'New York' } \\
\text { C.earnings }>300 \\
\quad \| \\
\text { C }\end{array}$ & $\begin{array}{l}\text { E.empl }=\text { C.cname } \\
\text { E.exp } \geq 20 \\
\text { E.age } \leq 65 \\
\text { C.loc }=\text { 'New York' } \\
\text { C.earnings > } 500 \\
\| \\
\text { D }\end{array}$ & $\mathrm{q}_{8}$ \\
\hline $\mathrm{q}_{6}$ & - & - & - & - & - & $\begin{array}{l}\text { E.empl = C.cname } \\
\text { E.exp } \geq 20 \\
\text { E.age } \leq 65 \\
\text { C.loc = 'New York' } \\
\text { C.eaernings > 300 } \\
\|_{8} \\
\mathrm{q}_{8}\end{array}$ & $\mathrm{q}_{8}$ \\
\hline $\mathrm{q}_{7}$ & - & - & - & - & - & - & $\mathrm{q}_{8}$ \\
\hline
\end{tabular}

(a)

$$
\begin{aligned}
& \mathcal{Q}_{1}=\{\} \\
& \mathcal{Q}_{2}=\left\{\text { output }\left(q_{1}\right), \text { output }(A)\right\} \\
& \mathcal{Q}_{3}=\left\{\text { output }\left(q_{1}\right), \text { output }(B)\right\} \\
& \mathcal{Q}_{4}=\left\{\operatorname{output}\left(q_{1}\right), \text { output }(A), \text { output }(B)\right\} \\
& \mathcal{Q}_{5}=\left\{\text { output }\left(q_{1}\right), \text { output }\left(q_{2}\right), \text { output }\left(q_{3}\right), \text { output }\left(q_{4}\right), \text { output }(C), \text { output }(D), \text { output }\left(q_{8}\right)\right\} \\
& \mathcal{Q}_{6}=\left\{\text { output }\left(q_{1}\right), \text { output }\left(q_{2}\right), \text { output }(B), \text { output }\left(q_{4}\right), \text { output }(C), \text { output }\left(q_{8}\right)\right\} \\
& \mathcal{Q}_{7}=\left\{\text { output }\left(q_{1}\right), \text { output }\left(q_{2}\right), \text { output }\left(q_{3}\right), \text { output }\left(q_{4}\right), \text { output }(D), \text { output }\left(q_{8}\right)\right\} \\
& \mathcal{Q}_{8}=\left\{\text { output }\left(q_{1}\right), \text { output }\left(q_{2}\right), \text { output }(B), \text { output }\left(q_{4}\right)\right\}
\end{aligned}
$$

Figure 8: (a) csq matrix corresponding to Figure 7 and (b) associated $\mathcal{Q}$ sets 


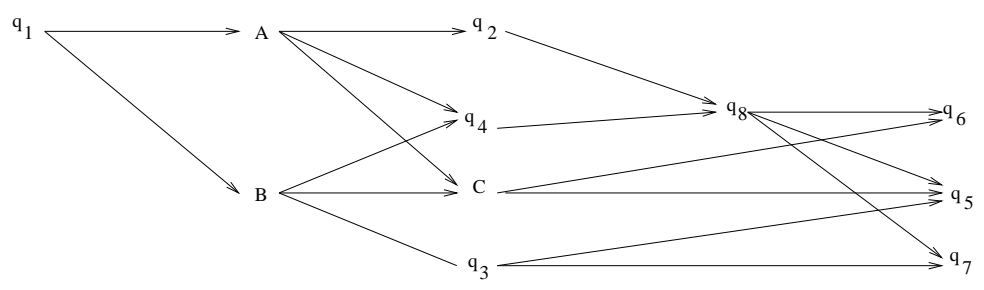

Figure 9: Relationship graph corresponding to Figure 7

We note that the elements in a number of sets has been considerably decreased due to Rule 1. For example, in $\mathcal{Q}_{6} 4$ of the 7 elements could be discarded. Since output $\left(q_{8}\right)$ is a subset of each element of $\mathcal{Q}_{8}$ and output $\left(q_{8}\right)$ can be used in processing $q_{6}$, output $\left(q_{1}\right)$, output $\left(q_{2}\right)$, output $(B)$, and output $\left(q_{4}\right)$ could be deleted from $\mathcal{Q}_{6}$.

Application of Rule 2 leads to the following results. Since $\mathcal{Q}_{5}$ and $\mathcal{Q}_{7}$ are the only sets satisfying to the condition of containing at least two queries that belong to the initial sequence $S$ and at least one common subquery that do not belong to $S$, we investigate for these sets whether common subqueries can be replaced by intersected queries or not. It appears that the intersected query of $q_{3}$ and $q_{8}$ is equal to the query corresponding to $D$. Since $q_{3}$ and $q_{8}$ are elements of $\mathcal{Q}_{5}$ as well as of $\mathcal{Q}_{7}, D$ can be discarded from $\mathcal{Q}_{5}$ and $\mathcal{Q}_{7}$. So, after application of Rules 1 and 2 the sets look as follows:

$\mathcal{Q}_{1}=\{\}$

$\mathcal{Q}_{2}=\left\{\operatorname{output}\left(q_{1}\right)\right.$, output $\left.(A)\right\}$

$\mathcal{Q}_{3}=\left\{\operatorname{output}\left(q_{1}\right)\right.$, output $\left.(B)\right\}$

$\mathcal{Q}_{4}=\left\{\operatorname{output}\left(q_{1}\right)\right.$, output $(A)$, output $\left.(B)\right\}$

$\mathcal{Q}_{5}=\left\{\right.$ output $\left(q_{3}\right)$, output $(C)$, output $\left.\left(q_{8}\right)\right\}$

$\mathcal{Q}_{6}=\left\{\right.$ output $(C)$, output $\left.\left(q_{8}\right)\right\}$

$\mathcal{Q}_{7}=\left\{\operatorname{output}\left(q_{3}\right)\right.$, output $\left.\left(q_{8}\right)\right\}$

$\mathcal{Q}_{8}=\left\{\right.$ output $\left(q_{2}\right)$, output $\left.\left(q_{4}\right)\right\}$

Step 3 of the algorithm results into the following csq matrix for $A, B$, and $C$.

\begin{tabular}{|c|c|c|}
\cline { 2 - 3 } \multicolumn{1}{c|}{} & $B$ & $C$ \\
\hline$A$ & $q_{1}$ & $A$ \\
\hline$B$ & - & $B$ \\
\hline
\end{tabular}

From this csq-matrix, we derive $\mathcal{Q}_{A}=\left\{q_{1}\right\}, \mathcal{Q}_{B}=\left\{q_{1}\right\}$, and $\mathcal{Q}_{C}=\{A, B\}$. Then, on the basis of the derived relationships between the queries, we can derive the graph of Figure 9 .

To decide whether an intermediate node will remain in the eventual graph or not, we apply Rule 3(a) and Rule 3(b), which is the key activity of step 4. For node $A$ holds that $S Q 1_{A}=\left\{q_{2}\right\}$, because the output of $q_{2}$ can be obtained by a selection on the output of $A$. For the other queries where $A$ can be used, i.e., $q_{3}, q_{4}$, and $C$, this is not the case. Let the cost to process the query corresponding to node $A$ be 1000 disk accesses ${ }^{2}$ and the processing cost to process $q_{2}$ whether or not using the output of $A$ is also 1000 . Then, node $A$ should

\footnotetext{
${ }^{2}$ This cost depends, of course, on database characteristics and the physical schema of the database. However, for illustrative purposes we have chosen some hypothetical cost values.
} 


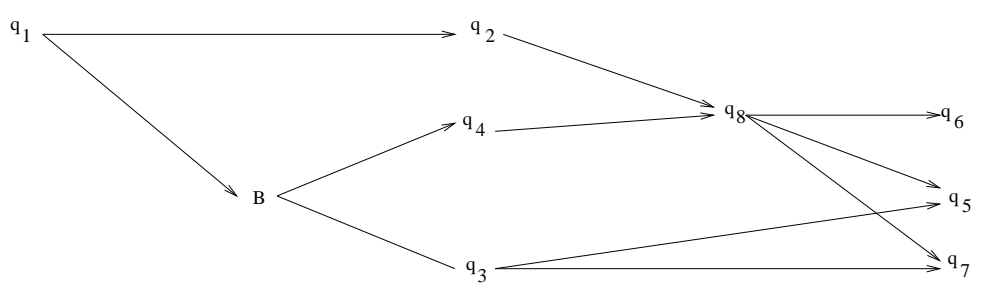

Figure 10: Reduced relationship graph

be removed from the graph according to Rule 3(a).

For node $B$ holds: $S Q 1_{B}=\left\{q_{3}, q_{4}, q_{5}, q_{6}\right\}$ and $S Q 2_{B}=\{\}$. Let the cost to process the query corresponding to $B$ be 1500 disk accesses, and the total cost to process queries $q_{3}, q_{4}, q_{5}$, and $q_{6}$ by using the output of $B$ be 800 disk accesses. The total cost to process the queries $q_{3}, q_{4}, q_{5}$, and $q_{6}$ without using the output of $B$ is 3000 disk accesses Then, $\operatorname{Cost}\left(S Q 1_{B}\right)+\operatorname{Cost}(B)=1500+800=2300<\operatorname{Cost}(S Q 1)=3000$. Thus, $B$ will be remain in the graph.

Let us assume that for node $\mathrm{C}$ it is decided that it should be discarded from the graph. Then, the graph of Figure 9 is reduced to Figure 10. We note that $S Q 1_{C}=\left\{q_{5}, q_{6}\right\}$.

\section{Conclusions \& FURTher RESEARCH}

As the variety of database applications grows rapidly, its impact on the performance requirements and the pattern of queries passed to the DBMS poses new research challenges. In some key database applications, such as data mining, a sequence of interdependent queries may be posed simultaneously to the DBMS. Optimizing such interdependent queries, called multiquery optimization, separately leads to performance that is far from optimal. This paper is devoted to the exploitation of the interdependencies between queries without re-development of complex query optimizers. We have presented an architecture for mult-query optimization that seamlessly fits into traditional optimization frameworks and is adaptive to emerging techniques. Based on this architecture, we have developed an algorithm that restructures a sequence of queries such that it can efficiently be processed by existing query optimizers. We have shown by means of a realistic case that our algorithm is promising in tackling the problem of multi-query optimization.

In the near future, we will implement the algorithm and connect it to the ORACLE DBMS. A thorough evaluation of this algorithm is another topic for the future.

REFERENCES

[AlRa94] Alsabbagh, J.R., Raghavan, V.V., Analysis of Common Subexpression Exploitation Models in Multiple Query Processing, in Proc. 10th Int. Conf. on Data Engineering, pp. 488-497.

[Choe95] Choenni, R., On the Automation of Physical Database Design, Ph.D. thesis, University of Twente, May 1995.

[ChSi96] Choenni, R., Siebes, A., Query Optimization to Support Data Mining, to appear.

[Fink82] Finkelstein, S., Common Expression Analysis in Database Applications, in Proc. of the 1982 ACM Int. Conf. on Management of Data, pp. 235-245.

[ElNa89] R. Elmasri, S.B. Navathe, Fundamentals of Database systems. The Benjamin/Cummings 
Publishing Company, California, USA, 1988.

[FiST88] Finkelstein, S., Schkolnick, M., Tiberio, P., Physical Database Design for Relational Databases, in ACM Trans. on Database Systems 13(1), 91-128.

[Grae93] Graefe, G., Query Evaluation Techniques for Large Databases, in ACM Computing Surveys 25(2), pp. 73-170.

[Jark84] Jarke, M., Common Subexpression Isolation in Multi Query Optimization, in Query Processing in Database Systems, Kim, W., Reinier, D., Batory, D., (eds), Springer Verlag, pp. 191-205.

[Kim84] Kim, W., Global Optimization of Relational Queries: A First Step, Multi Query Optimization, in Query Processing in Database Systems, Kim, W., Reinier, D., Batory, D., (eds), Springer Verlag, pp. 206-216.

[LaYa85] Larson, P-A., Yang, H.Z., Computing Queries from Derived Relations, in Proc. 11th Int. Conf. on Very Large Data Bases, pp. 259-269.

[Rous82a] Roussopoulos, N., View Indexing in Relational Databases, in ACM Trans. on Database systems 7(2), pp. 258-290.

[Rous82b] Roussopoulos, N., The Logical Access Path Schema of a Database, in IEEE Trans. on Software Engineering 8(6), pp. 562-573.

[RoHu80] Rosenkrantz, D.J., Hunt, H.B., Processing Conjunctive Predicates and Queries, in Proc. 6th Int. Conf. on Very Large Data Bases, pp. 64-72.

[SACL79] Selinger, P., Astrahan, M.M., Chamberlin, D.D., Lorie, R.A., Price, T.G., Access Path Selection in a Relational Database Management System, in Proc. of the 1979 ACM Int. Conf. on Management of Data.

[Sell88] Sellis, T.K., Multiple-Query Optimization, in ACM Trans. on Database systems 13(1), pp. 23-52.

[Swam89] Swami, A., Optimization of Large Join Queries: Combining Heuristics and Combinatorial Approach, in Proc. of the 1989 ACM Int. Conf. on Management of Data, pp. 367-376.

[Ullm89] J.D. Ullman, Principles of Database and Knowledge-Base Systems, Vol.2: The New Technologies, Computer Science Press, New York, USA.

[Yao77] S.B. Yao, Approximating Block Accesses in Database Organizations, in Comm. of the ACM 32(5), pp. 260-261. 